\title{
Lymphovascular invasion predicts disease-specific survival in node-negative esophageal squamous cell carcinoma patients after minimally invasive esophagectomy
}

\author{
Ying-Jian Wang, Xiao-Long Zhao, Kun-Kun Li, Xue-Hai Liu, Tao Bao, Wei Guo \\ Department of Thoracic Surgery, Daping Hospital, Army Medical University, Chongqing, China
}

Videosurgery Miniinv 2022; 17 (2): 309-316

DOI: https://doi.org/10.5114/wiitm.2021.112679

\begin{abstract}
Introduction: Lymphovascular invasion (LVI) is reported to be a potential prognostic predictor in esophageal squamous cell carcinoma (ESCC) patients.

Aim: To investigate the prognostic value of LVI in ESCC node-negative patients after minimally invasive esophagectomy (MIE).

Material and methods: 1406 consecutive ESCC patients who underwent MIE were reviewed retrospectively. After exclusion, 880 patients were enrolled, and 298 node-negative patients were used for the further analysis. The Kaplan-Meier method was used to examine the survival difference. Univariate and multivariate analyses were performed to identify prognostic predictors.

Results: LVI was observed in $29.4 \%$ of all patients. Totally, the proportion of LVI was increased with advanced T $(p<0.01)$ and $N(p<0.01)$ stage and poor tumor differentiation $(p<0.01)$. In the node-negative patients, a similar result was obtained in T stage $(p=0.0252)$ and tumor differentiation $(p=0.0080)$. In survival analysis, the disease-specific survival (DSS) ( $p=0.0146)$ rate was significantly lower in node-negative patients with LVI than in those without. The difference was absent when calculating disease-free survival (DFS) ( $p=0.0796)$. Additionally, the presence of LVI was associated with lower DSS $(p=0.0187)$ but not DFS $(p=0.0785)$ in univariate analysis in node-negative patients. Moreover, in multivariate Cox regression analysis, the presence of LVI was identified as an independent prognostic factor only in DSS $(p=0.0496)$ but not in DFS $(p=0.5670)$ in node-negative patients.

Conclusions: LVI is associated with shorter DSS and an independent prognostic factor in ESCC node-negative patients after MIE.
\end{abstract}

Key words: lymphovascular invasion, esophageal squamous cell carcinoma, minimally invasion esophagectomy, survival, prognostic value.

\section{Introduction}

Esophageal cancer is an aggressive malignant tumor worldwide and patients often present with an advanced disease stage, encompassing primary advanced tumor and metastatic lymph nodes. Lymph node metastasis is known to be one of the most important risk factors for prognosis in patients with esophageal cancer. The prognosis gets progressively worse with increasing number of involved lymph nodes. However, in parts of patients without lymph nodes metastasis still has a high recurrence rate even after curative resection. It indicates that using lymph node status alone as the major pathological feature to assess the prognosis is less accurate 
than in combination with other factors in patients without lymph node metastasis. Therefore, additional modifications with other pathological features should be proposed to achieve higher accuracy of staging, which is helpful in guiding postoperative treatment and follow-up.

Recently, lymphovascular invasion (LVI) was identified as an important pathological feature, which indicates the presence of tumor cells within the lumen of the vein and lymphatics, in predicting poor prognosis in multiple kinds of cancers [1-5]. In the process of lymph node metastasis and the systemic dissemination of cancer cells, LVI has been demonstrated to be an essential step and considered to increase the risk for micrometastasis in localized carcinoma [6]. More and more evidence has been provided to demonstrate the relationship between LVI and unsatisfactory prognostication of solid tumors; however, the role of LVI in prognosis of esophageal carcinoma has not been adequately investigated. Most previous studies were limited by the small sample size, insufficient lymph nodes dissection, inadequate surgical resection and heterogeneity of pathological types, tumor stages, surgical types and perioperative treatments. The limitations are mirrored by significant variants in outcomes worldwide.

\section{Aim}

In this study, we aimed to evaluate the prognostic value of LVI in node-negative patients with esophageal squamous cell carcinoma (ESCC) who underwent minimally invasive esophagectomy (MIE) without any preoperative adjuvant therapy.

\section{Material and methods}

\section{Patients}

This retrospective study included 1406 consecutive patients who underwent esophagectomy between September 2009 and May 2020 at the Department of Thoracic Surgery, Daping Hospital, Army Medical University, Chongqing, China for ESCC. To obtain unbiased data, we excluded patients with neoadjuvant regimens, including chemotherapy and chemoradiotherapy, noncurative (R1 or R2) resection, advanced T stage (T4), and lymph node harvest less than 15 after lymphadenectomy. After surgery, 68 patients were lost to follow-up, and 24 patients died from postoperative complications within 30 days. Finally, 880 patients were enrolled for further studying, including 298 node-negative patients (Figure 1). Clinicopathologic data of all patients were routinely collected. After surgery, all patients were followed up regularly in the outpatient clinic every 3 months during the first 2 years, every 6 months until the fifth year, and then annually. A diagnosis of recurrence was made by using either pathologic or radiologic confirmation. The primary endpoint was disease-free survival (DFS), which was calculated from the date of surgery to the date of first recurrence or metastasis of cancer. The secondary end point was disease-specific survival (DSS), which was calculated from the date of surgery to the date of death due to a specific disease. Written consent

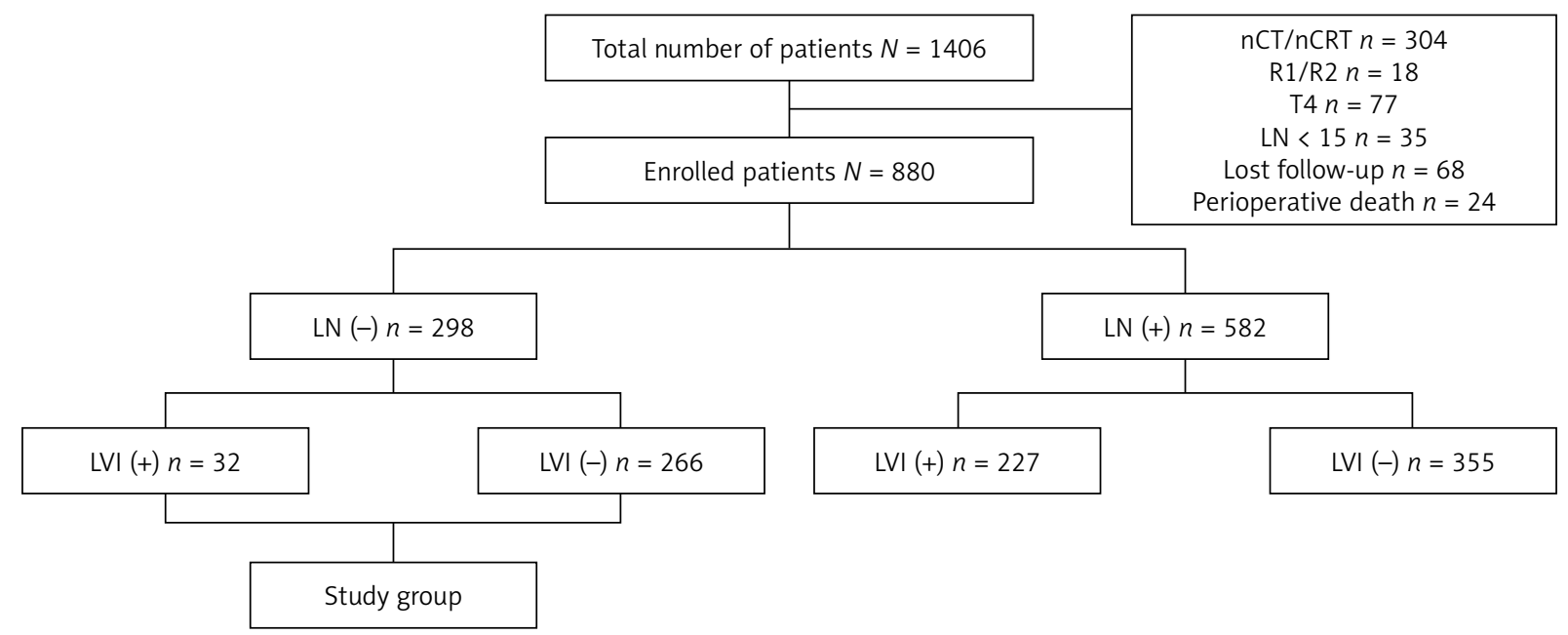

Figure 1. Study enrollment 
was obtained from the patients associated with this study, and the study was approved by the Ethics Committee of Daping Hospital, Army Medical University, Chongqing, China.

\section{Surgery}

All operations were performed under double-lumen intubation. The method used to perform it was described previously [7]. Thoracoscopic esophagectomy was performed with the patient in the semiprone position and four trocars were inserted: a $30^{\circ}$ $12-\mathrm{mm}$ thoracoscope was inserted through a $12-\mathrm{mm}$ port in the eighth intercostal space (ICS) at the posterior axillary line (PAL); a 5-mm port was placed in the sixth ICS immediately cephalic and posterior to the tip of the scapula; a $12-\mathrm{mm}$ port and $5-\mathrm{mm}$ port were placed halfway between the spine and the original fourth and sixth intercostal port separately. The surgeon and camera operator stood at the patient's right side, and the video monitor was positioned on the patient's left side. Esophageal mobilization and dissection were performed essentially in the same manner as in open surgery; the surgery was begun by cauterizing the mediastinal pleura overlying the anterior aspect of the esophagus and mobilizing the esophagus from the hilum and the pericardium. Mobilization extended to the level of the azygous vein, which was skeletonized and ligated with 10-mm Liga clips. With electrocautery, the parietal pleura posterior to the esophagus was opened from the level of the azygous vein to the crus. After dissecting the esophagus and mediastinal lymph nodes, the thoracic duct was routinely mass ligated immediately above the diaphragmatic hiatus. After completing the thoracic procedure, the patient was rotated to a supine position, with the neck extended and turned toward the right. During the laparoscopic procedure, the entire greater curvature of the stomach was first mobilized, followed by division of the omentum. Then, the lymph nodes were dissected from the common hepatic artery to the left gastric artery, including the proximal splenic artery and the celiac artery. All these nodes, including the common hepatic nodes (station $18^{\text {th }}$ lymph node), the splenic nodes (station $19^{\text {th }}$ lymph node), the left gastric nodes (station $17^{\text {th }}$ lymph node), and the celiac nodes (station $20^{\text {th }}$ lymph node) were dissected en bloc. The left gastric vessels were divided after ligation with Hem-o-lok clips. Lastly, the right and left crura of the diaphragm were dissected, and the abdominal cavity was linked to the mediastinum. In the last step, a $5-\mathrm{cm}$ oblique incision along the anterior border of the left sternocleidomastoid muscle was made. The cervical esophagus was mobilized and transected. After placing the anastomat component in the proximal end, the distal end was connected with a belt. Subsequently, the subxiphoid incision was enlarged to $3 \mathrm{~cm}$, the esophagus and stomach were pulled out, and the gastric tube was formatted with a stapler, with a width of $3 \mathrm{~cm}$ and a length of $35 \mathrm{~cm}$. The gastric tube was pulled up to the left neck through the posterior mediastinum. After completing the esophagogastric anastomosis with a circular stapler, we closed the gastric stump with a stapler and placed an $18 \mathrm{Fr}$ drainage tube in the cervical incision.

\section{Histopathologic examination}

The resection specimens were fixed with formaldehyde (4\%) for $24 \mathrm{~h}$. The complete tumor was cut in slices of $0.5 \mathrm{~cm}$. All lymph nodes were dissected and analyzed. The tissue was paraffin embedded and stained with hematoxylin, eosin and van Gieson stain. All specimens were classified by two experienced pathologists according to the AJCC criteria.

LVI was defined as tumor cells spreading through the lymphatic vessels, i.e., carcinoma cells floating within the endothelial-lined space $[1,6]$. The histological diagnosis of LVI was established according to the following criteria: (i) the presence of tumor cells within the lymphatic or vascular space and (ii) evidence of tumor cells attached to the vascular wall. Immunohistochemical (IHC) staining for endothelial cells was performed routinely, keeping with the standard practice of pathology. CD31, CD34, and D2-40 were used to detect LVI.

\section{Statistical analysis}

The categorical variables were compared between the LVI-positive and LVI-negative groups using Fisher's exact test. The survival curve was depicted using the Kaplan-Meier method and compared using the log-rank test. Univariate and multivariate survival analysis was performed using the Cox proportional hazard regression model. Statistical significance was set at $p<0.05$, and all tests were two-sided. Statistical analyses were carried out using MedCalc 12 (MedCalc Software, Ostend, Belgium). 


\section{Results}

The clinicopathologic characteristics of total and LVI-positive patients with ESCC are summarized in Table I. In total, the presence of LVI was observed in $29.4 \%$ of the patients (259/880), whereas $70.6 \%$ of the patients $(621 / 880)$ had no evidence of LVI. We found that 3.2\% (5/154), 15.4\% (32/208) and $42.9 \%$ (222/518) of patients had stages T1, T2 and T3 with
$L V I$, respectively. The difference was highly significant $(p<0.01)$. It was also reflected by the percentage of the presence LVI in lymph node metastasis (N0 10.7\% (32/298), N1 32.3\% (130/403), N2 46.2\% (67/145), N3 88.2\% (30/34)), which was also significant $(p<0.01)$. Regarding the tumor grade, we found that $14.2 \%(35 / 247)$ of the well-differentiated (G1), $29.8 \%(134 / 450)$ of the moderately differentiated

Table I. Clinicopathologic characteristics of the patients

\begin{tabular}{|c|c|c|c|c|c|c|}
\hline \multirow[t]{2}{*}{ Variable } & \multicolumn{2}{|c|}{ Total } & \multirow[t]{2}{*}{$P$-value } & \multicolumn{2}{|c|}{ LN - } & \multirow[t]{2}{*}{$P$-value } \\
\hline & No. & $L V I+(\%)$ & & No. & $L \mathrm{LI}+(\%)$ & \\
\hline Total & 880 & 259 (29.4\%) & & 298 & $32(10.7 \%)$ & \\
\hline Sex: & & & 0.1881 & & & 0.7783 \\
\hline Men & 678 & $192(28.3 \%)$ & & 214 & $23(10.7 \%)$ & \\
\hline Women & 202 & 67 (33.2\%) & & 84 & 9 (10.7\%) & \\
\hline Age: & & & 0.3481 & & & 0.1017 \\
\hline$>60$ years & 583 & $178(30.5 \%)$ & & 208 & $18(8.7 \%)$ & \\
\hline$\leq 60$ years & 297 & 81 (27.2\%) & & 90 & 14 (15.6\%) & \\
\hline Smoking status: & & & 0.0567 & & & 0.0896 \\
\hline Yes & 601 & 189 (31.4\%) & & 242 & 22 (9.1\%) & \\
\hline No & 279 & $70(25.1 \%)$ & & 56 & 10 (17.9\%) & \\
\hline BMI [kg/m²]: & & & 0.1507 & & & 0.0656 \\
\hline$<18.5$ & 142 & 41 (28.9\%) & & 25 & $3(12 \%)$ & \\
\hline $18.5-24$ & 624 & 193 (39.9\%) & & 236 & 21 (8.9\%) & \\
\hline$>24$ & 114 & 25 (21.9\%) & & 37 & $8(21.6 \%)$ & \\
\hline T classification: & & & $<0.01$ & & & 0.0252 \\
\hline pT1 & 154 & $5(3.2 \%)$ & & 47 & $1(2.1 \%)$ & \\
\hline pT2 & 208 & $32(15.4 \%)$ & & 81 & $6(7.4 \%)$ & \\
\hline pT3 & 518 & $222(42.9 \%)$ & & 170 & 25 (14.7\%) & \\
\hline N classification: & & & $<0.01$ & & & N/A \\
\hline pNO & 298 & $32(10.7 \%)$ & & 298 & 32 (100\%) & \\
\hline pN1 & 403 & $130(32.3 \%)$ & & & & \\
\hline pN2 & 145 & $67(46.2 \%)$ & & & & \\
\hline pN3 & 34 & 30 (88.2\%) & & & & \\
\hline Grade: & & & $<0.01$ & & & 0.0080 \\
\hline G1 & 247 & 35 (14.2\%) & & 109 & $5(4.6 \%)$ & \\
\hline G2 & 450 & $134(29.8 \%)$ & & 147 & $18(12.2 \%)$ & \\
\hline G3 & 183 & $90(49.2 \%)$ & & 42 & 9 (21.4\%) & \\
\hline Location: & & & 0.0854 & & & 0.2940 \\
\hline Upper & 203 & 65 (32.0\%) & & 47 & $2(4.3 \%)$ & \\
\hline Middle & 561 & $173(30.8 \%)$ & & 175 & 21 (12\%) & \\
\hline Lower & 116 & 21 (18.1\%) & & 76 & 9 (11.8\%) & \\
\hline
\end{tabular}

LVI - lymphovascular invasion, BMI - body mass index, No. - number, LN - lymph node. 
(G2) and $49.2 \%(90 / 183)$ of the poorly differentiated (G3) patients showed a positive LVI in the tumor $(p<0.01)$. No significant difference was observed according to sex, age, smoking status, BMI, or tumor location. Similarly, in patients without lymph node metastasis, only $\mathrm{T}$ stage $(p=0.0252)$ and tumor grade $(p=0.0080)$ exhibited a significant difference in different lymphovascular invasion status.

A total of 582 patients were alive at the time of analysis in all 880 cases. The DFS in the entire study cohort was $62.4 \%$, with a median survival time of 48 months, and the DSS was $66.1 \%$, with a median survival time of 61 months. In the subgroup of node-negative patients, the presence of LVI had a significantly lower DSS than in those without

A

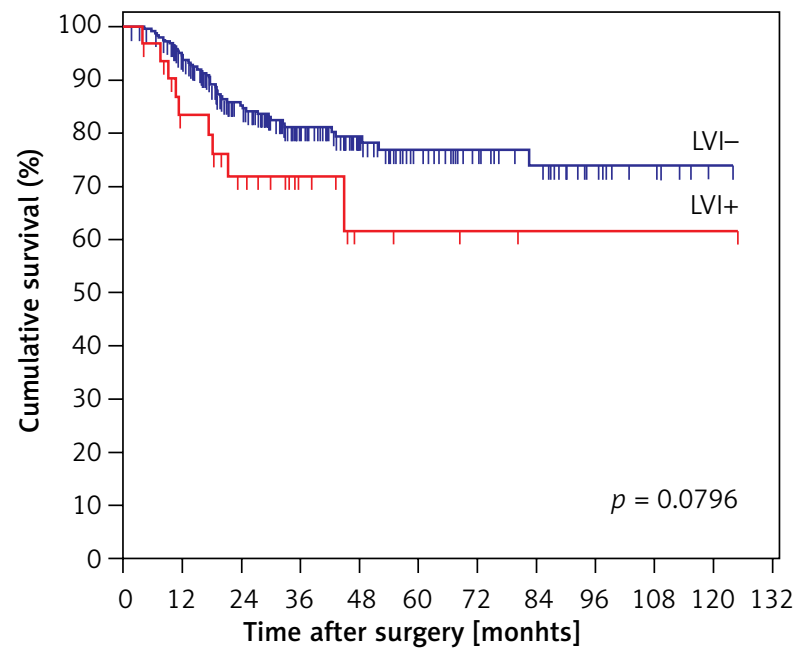

$(p=0.0149)$ (Figure 2 B). The difference was absent when the survival analysis was used for calculating DFS ( $p=0.0796)$ (Figure 2 A).

In univariate analysis, poorly differentiated grade and advanced pathologic $T$ stage were identified to be significantly associated with poor DFS and DSS in patients without lymph node metastasis. However, the presence of LVI exhibited prognostic nature only in DSS $(p=0.0189)$ but not in DFS $(p=0.0785)$ in patients without lymph node metastasis (Table II). As the same result as in univariate analysis, the presence of LVI in node-negative patients was identified as an independent prognostic factor only in DSS $(p=0.0496)$ but not in DFS $(p=0.5670)$ in multivariate Cox regression analysis (Table III).

\section{B}

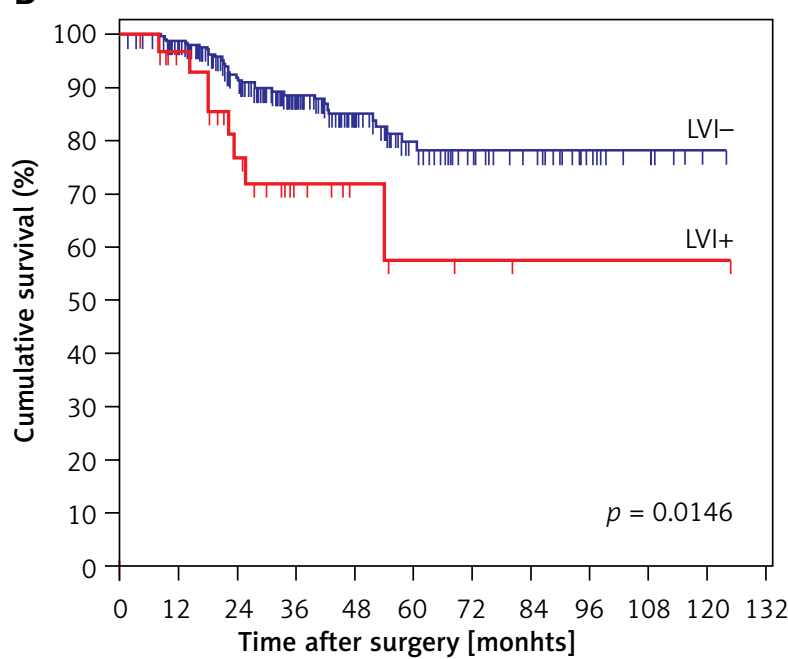

Figure 2. Kaplan-Meier curves of survival rates for patients without lymph node metastasis (NO stage) in this study. A - Disease-free survival (DFS) rates for patients with or without lymphovascular invasion (LVI). B - Disease-specific survival (DSS) rates for patients with or without lymphovascular invasion (LVI)

Table II. Univariate analysis of prognostic factors for DFS and DSS in patients without lymph node metastasis

\begin{tabular}{|lcccccc|}
\hline Variables & \multicolumn{3}{c}{ DFS } & \multicolumn{2}{c|}{ DSS } \\
\cline { 2 - 7 } & $\mathrm{HR}$ & $95 \% \mathrm{Cl}$ & $P$-value & $\mathrm{HR}$ & $95 \% \mathrm{Cl}$ & $P$-value \\
\hline LVI & 0.4598 & $0.1943-1.0879$ & 0.0785 & 0.6465 & $0.2091-1.9992$ & 0.0187 \\
\hline pT classification & 0.5645 & $0.3342-0.9537$ & 0.0335 & 0.5563 & $0.2799-1.1056$ & 0.0359 \\
\hline Grade & 0.5647 & $0.3488-0.9144$ & 0.0208 & 0.4413 & $0.2228-0.8741$ & 0.0196 \\
\hline Age & 1.8027 & $0.7294-4.4553$ & 0.2041 & 2.4106 & $0.6960-8.3490$ & 0.1672 \\
\hline BMI & 1.3714 & $0.6913-2.7211$ & 0.3686 & 1.1364 & $0.3588-2.1577$ & 0.7808 \\
\hline Sex & 1.157 & $0.5023-2.6652$ & 0.7333 & 1.1116 & $0.3813-3.2411$ & 0.8471 \\
\hline Smoking status & 1.7382 & $0.8232-3.6697$ & 0.1491 & 1.4859 & $0.5673-3.8910$ & 0.4226 \\
\hline
\end{tabular}


Table III. Multivariate Cox regression analysis of prognostic factors for DFS and DSS in patients without lymph node metastasis

\begin{tabular}{|lcccccc|}
\hline Variables & \multicolumn{3}{c}{ DFS } & \multicolumn{2}{c|}{ DSS } \\
\cline { 2 - 7 } & $\mathrm{HR}$ & $95 \% \mathrm{Cl}$ & $P$-value & $\mathrm{HR}$ & $95 \% \mathrm{Cl}$ & $P$-value \\
\hline LVI & 0.7940 & $0.3537-1.7822$ & 0.5670 & 0.4536 & $0.2061-0.9985$ & 0.0496 \\
\hline pT classification & 0.2675 & $0.0772-0.9262$ & 0.0384 & 0.5563 & $0.2799-1.1056$ & 0.0459 \\
\hline Grade & 0.5898 & $0.3618-0.9613$ & 0.0351 & 0.4073 & $0.1706-0.9729$ & 0.0442 \\
\hline Age & 1.5693 & $0.5933-4.1506$ & 0.3663 & 1.9964 & $0.5083-7.8404$ & 0.3244 \\
\hline BMI & 1.7455 & $0.8711-3.4977$ & 0.1181 & 1.5033 & $0.6202-3.6443$ & 0.3692 \\
\hline Sex & 1.441 & $0.6183-3.3583$ & 0.3998 & 2.8474 & $0.4506-9.5602$ & 0.3512 \\
\hline Smoking status & 1.6984 & $0.8344-3.4578$ & 0.1461 & 2.9691 & $0.7349-11.9904$ & 0.1285 \\
\hline
\end{tabular}

DFS - disease-free survival, DSS - disease-specific survival, HR - hazard ratio, Cl - confidence interval, LVI - lymphovascular invasion, BMI - body mass index.

\section{Discussion}

Lymph node metastasis has been widely accepted as an important negative prognostic predictor in ESCC patients; however, a significant fraction of node-negative patients still suffer from disease recurrence even after radical surgery. Survival rates may obscure the relationship between outcome and prognostic indicators in this group of patients. Therefore, additional prognostic parameters should be added to the current TNM system in order to establish a comprehensive appraisal system for ESCC patients without lymph node metastasis. Recently, LVI was identified as an important prognostic factor in many kinds of malignant tumors [1-5]. In our study, the presence of LVI was observed in $29.4 \%$ of all patients, and a correlation was found between presence of LVI and poor overall survival rate in node-negative patients, which was in accordance with some previous studies [8-12]. Additionally, multivariate analysis demonstrated that presence of LVI was an independent prognostic factor in overall survival for ESCC patients without lymph node metastasis.

LVI is a histopathological feature that indicates the presence of tumor cells within the lumen of vein and lymphatics, which has been demonstrated as a negative prognostic parameter in many kinds of solid tumors. However, the specific role of LVI in patients with ESCC remains uncertain, and the available evidence is often conflicting. In some previous studies, the presence of LVI indicated a worse outcome in survival analysis [13-16]; on the other hand, some researchers considered that more evidence should be obtained to demonstrate the prognostic value of LVI in esophageal carcinoma $[17,18]$. To our knowledge, one reason for the contradictory conclusions might be that the lymph node status was not considered in some previous studies. It is known that a survival advantage is conferred by the absence of metastatic lymph nodes, which is strongly associated with survival benefit. The prognostic value of LVI may be affected by nodal status; therefore, only negative lymph node patients were included in our study to eliminate the effect of nodal status.

Although controversy still exists whether LVI is an independent prognostic factor in patients with ESCC, more and more evidence has been provided to demonstrate its adverse prognostic nature independent of clinicopathological features [19-22]. In agreement with these published studies, we found that LVI was an independent prognostic factor in ESCC patients without lymph node metastasis in multivariable analysis. On the other hand, some researchers found that LVI was associated with an unfavorable survival rate, but it was not independently significant based on multivariate analysis $[13,17,18]$. Indeed, these previous cohorts had some limitations. Some studies included patients with both ESCC and adenocarcinoma in various segments of the population. In addition, patients without adequate lymph node dissection which affected the accuracy of postoperative diagnosis were included. Moreover, some patients enrolled had received $\mathrm{R} 1$ or $\mathrm{R} 2$ resection and even with distant metastatic tumors. Furthermore, different preoperative treatments, such as neoadjuvant chemotherapy or chemoradiotherapy, were performed in these studies. Hence, we conducted the current homogeneous cohort study in an attempt to decrease selective biases. 
Although we have demonstrated the prognostic value of LVI in ESCC patients without lymph node metastasis, the mechanism of how LVI influences the dissemination of cancer cells from the primary tumor to regional lymph nodes is still unclear. Generally, one of the earliest steps in the metastatic cascade is LVI, which indicates the penetration of tumor cells into lymph vessels in and around the primary tumor. Therefore, emboli of tumor cells in lymphatic vessels are considered to be the morphological correlation of malignant metastasis to loco-regional and/or distant lymph nodes [23]. Nevertheless, the mechanism of the process is still unclear. In the future, we will attempt to conduct research to elucidate the mechanism.

The current study still had limitations, one of which is that the sample size was not large enough. Another one is that all the specimens were evaluated by hematoxylin and eosin staining examinations without special biomarkers. Trials with larger cohorts will be required to enhance the strength of our results in future.

\section{Conclusions}

The results of this study suggested that LVI is associated with shorter disease-specific survival and an independent prognostic factor in ESCC patients without lymph node metastasis.

\section{Acknowledgments}

The authors would like to thank the Department of Pathology, Daping Hospital, Army Medical University, China.

Ying-Jian Wang and Xiao-Long Zhao contributed equally to this work, and both should be considered first author.

\section{Conflict of interest}

The authors declare no conflict of interst.

\section{References}

1. Lotan Y, Gupta A, Shariat SF, et al. Lymphovascular invasion is independently associated with overall survival, cause-specific survival, and local and distant recurrence in patients with negative lymph nodes at radical systectomy. J Clin Oncol 2005; 23: 6533-9.

2. Lee AH, Pinder SE, Macmillan RD, et al. Prognostic value of lymphovascular invasion in women with lymph node negative invasive breast carcinoma. Eur J Cancer 2006; 42: 357-62.
3. Woo CS, Silberman H, Nakamura SK, et al. Lymph node status combined with lymphovascular invasion creates a more powerful tool for predicting outcome in patients with invasive breast cancer. Am J Surg 2002; 184: 337-40.

4. Reuter VE. Lymphovascular invasion as an independent predictor of recurrence and survival in node-negative bladder cancer remains to be proven. J Clin Oncol 2005; 23: 6450-1.

5. Yanagawa N, Shiono S, Abiko M, et al. Prognostic impact and initial recurrence site of lymphovascular and visceral pleural invasion in surgically resected stage I non-small-cell lung carcinoma. Eur J Cardiothoracic Surg 2013; 44: e200-6.

6. Kikuchi E, Margulis V, Karakiewicz PI, et al. Lymphovascular invasion predicts clinical outcomes in patients with node-negative upper tract urothelial carcinoma. J Clin Oncol 2009; 27: 612-8

7. Guo W, Zhao YP, Jiang YG, et al. Prevention of postoperative chylothorax with thoracic duct ligation during video-assisted thoracoscopic esophageactomy for cancer. Surg Endosc 2012; 26: 1332-6.

8. Brucher BL, Stein HJ, Werner W, et al. Lymphatic vessel invasion is an independent prognostic factor in patients with a primary resected tumor with esophageal squamous cell carcinoma. Cancer 2001; 92: 2228-33.

9. Kim T, Grobmyer SR, Smith R, et al. Esophageal cancer - the five year survivors. J Surg Oncol 2011; 103: 179-83.

10. Reid TD, Chan DSY, Roberts SA, et al. Prognostic significance of circumferential resection margin involvement following oesophagectomy for cancer and the predictive role of endoluminal ultrasonography. Br J Cancer 2012; 107: 1925-31.

11. Wang LS, Chow KC, Chi KH, et al. Prognosis of esophageal squamous cell carcinoma: analysis of clinicopathological and biological factors. Am J Gastroenterol 1999; 94: 1933-40.

12. Wang LS, Chow KC, Wu YC, et al. Inverse expression of dihydrodiol dehydrogenase and glutathione-S-transferase in patients with esophageal squamous cell carcinoma. Int J Cancer 2004; 111: 246-51.

13. Sugimachi K, Matsuura $\mathrm{H}$, Kai $\mathrm{H}$, et al. Prognostic factors of esophageal carcinoma: univariate and multivariate analyses. J Surg Oncol 1986; 31: 108-12.

14. Schoppmann SF, Jesch B, Zacherl J, et al. Lymphangiogenesis and lymphovascular invasion diminishes prognosis in esophageal cancer. Surgery 2013; 153: 526-34.

15. Zhu CM, Ling YH, Xi SY, et al. Prognostic significance of the $p N$ classification supplemented by vascular invasion for esophageal squamous cell carcinoma. PLoS One 2014; 9: e96129.

16. Wang S, Chen X, Fan J, et al. Prognostic significance of lymphovascular invasion for thoracic esophageal squamous cell carcinoma. Ann Surg Oncol 2016; 23: 4101-9.

17. Sliwa B, Szelachowski P, Strutynska-Karpinska M. An analysis of the impact of clinico-pathological features on long-term results following esophagectomy due to squamous cell carcinoma of the thoracic esophagus. Adv Clin Exp Med 2013; 22: 369-75.

18. Zhu Z, Yu W, Li H, et al. Nodal skip metastasis is not a predictor of survival in thoracic esophageal squamous cell carcinoma. Ann Surg Oncol 2013; 20: 3052-8.

19. Chen WH, Huang YL, Chao YK, et al. Prognostic significance of lymphovascular invasion in patients with esophageal squa- 
mous cell carcinoma treated with neoadjuvant chemoradiotherapy. Ann Surg Oncol 2015; 22: 338-43.

20. Lagarde SM, Phillips AW, Navidi M, et al. The presence of lymphovascular and perineural infiltration after neoadjuvant therapy and oesophagectomy identifies patients at high risk for recurrence. Br J Cancer 2015; 113: 1427-33.

21. Huang QY, Luo KJ, Chen C, et al. Identification and validation of lymphovascular invasion as a prognostic and staging factor in node-negative esophageal squamous cell caicinoma. J Thor Oncol 2016; 11: 483-592.

22. Wang Z, Chen P, Wang F, et al. Lymphovascular invasion as an independent prognostic indicator in radically resected thoracic esophageal squamous cell carcinoma. Thorac Cancer 2019; 10: 150-5.

23. Van den Eynden G, Van der Auwera I, Van Laere S, et al. Distinguishing blood and lymph vessel invasion in breast cancer: a prospective immunohistochemical study. Br J Cancer 2006; 94: 1643-9.

Received: 10.09.2021, accepted: 25.10.2021. 